\title{
An Improved Very Low-Power Electrode Contact Impedance Monitor
}

\author{
Martin J Burke ${ }^{1, a}$, Cormac Molloy ${ }^{1}$ and Helge Fossan ${ }^{2}$ \\ ${ }^{1}$ Dept. of Electronic and Electrical Engineering, Trinity College, University of Dublin, College Green, Dublin 2, Ireland. \\ ${ }^{2}$ Laerdal Medical AS, P.O. Box 337, Tanke Svilandsgate 30, N-4002 Stavanger, Norway.
}

\begin{abstract}
This paper reports the design improvement of a circuit for monitoring the quality of contact of un-gelled stainless steel electrodes with a patient's skin in the measurement of the ECG. The circuit is intended to be incorporated into a heart rate monitor used prior to and during childbirth to measure the mother's heart rate. The revised design uses commercially available low-power technology to implement the circuit with a reduction in the power consumption by a factor of 20 over a previous design [1]. The circuit measures the quality of contact of electrode impedances up to $100 \mathrm{k} \Omega$ and gives a visual indication when the contact impedance of either electrode is above this level. The impedance is measured using a signal injected through the electrodes via the input impedance of the amplifier at a frequency of $5 \mathrm{kHz}$. The resulting signal present at the amplifier input at this frequency is then extracted and processed before threshold detection of the signal level which is used to indicate whether or not the quality of contact of the patient's fingers with the electrodes is acceptable for the purposes of heart rate measurement.
\end{abstract}

\section{Introduction}

The Safer Births Programme [2], sponsored by the Norwegian Research Council as part of a larger World Health Organization initiative [3], is an action plan aimed at eliminating preventable deaths of infants at birth, in developing countries. Many maternity hospitals in these locations are understaffed and are often forced to rely on midwives and nurses alone to contend with the clinical complications that arise around childbirth, without access to the assistance or equipment needed to deal with them. Few places have sufficient equipment for fetal monitoring such that fetuses in distress can be left unattended, adding to the burden of birth asphyxia and stillbirth. Many of the infants born may not be breathing correctly and have pulses which are difficult or impossible to detect manually. On occasions these infants can be misclassified as stillborn, when their hearts are still functioning and they could be resuscitated with suitable equipment. Laerdal Medical AS, based in Norway, designs and markets resuscitation equipment for use in the scenarios described above. One such product is the Moyo unit shown in Figure 1. This unit is a heart rate monitor which uses an ultrasonic transducer placed on the mother's abdomen to detect the infant's heart beat and indicates the fetal heart rate on a LCD. One common problem with ultrasonic heart rate monitors is that when the infant's heart beat is difficult to detect the transducer often picks up the mother's heart rate in error [4]. In order to overcome this problem the Moyo unit incorporates an ECG amplifier which is used to measure the mother's heart rate by way of an ECG signal picked up from her hands. This heart rate value is then indicated

\footnotetext{
${ }^{a}$ Corresponding author: mburke@tcd.ie
}

separately on the LCD. The Moyo unit is held by the mother giving birth who presses her fingers onto the stainless steel electrodes of the unit, which then detects her ECG and measures and displays her heart rate. The values of the two heart rates can then be compared by the midwife and used to discern whether or not the ultrasonic transducer is measuring the infant's heart rate reliably or the mother's heart rate in error. As the mother may be in distress during childbirth, the problem arises of ensuring that she maintains an adequate grip on the monitor to allow her ECG to be detected reliably.

It is intended to implement a mechanism in the Moyo unit which will verify that the electrical contact of the mother's fingers with the stainless steel un-gelled electrodes is acceptable. This is done by measuring the contact impedance of the skin-electrode interface at each electrode to assess whether or not the mother has pressed her fingers properly onto the electrodes. Surface contact

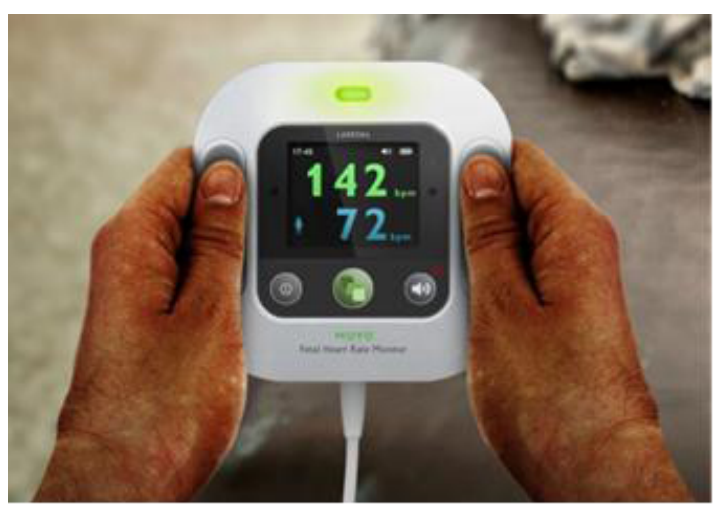

Figure 1. The MOYO Unit in Use 


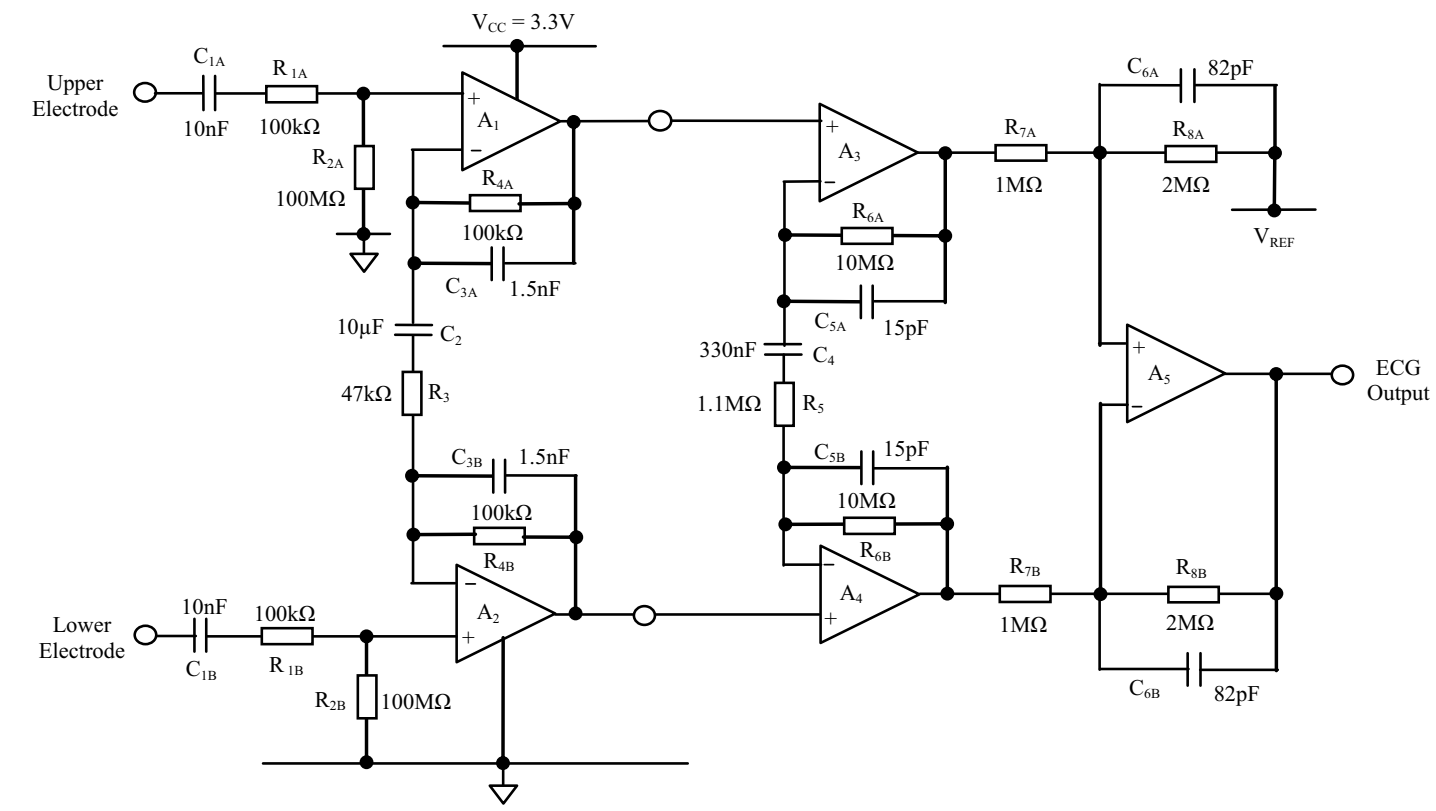

Figure 2. Schematic Diagram of the Original ECG Amplifier

impedance of each electrode is measured independently and a visual alarm is activated when the state of contact of either electrode is unsatisfactory for the purposes of reliable ECG measurement.

\section{Background}

\subsection{Existing ECG Amplifier}

The schematic diagram of the ECG amplifier originally used in the Moyo unit is shown in Figure. 2. This is a three-stage instrumentation ECG amplifier adapted from an earlier design by Burke \& Gleeson [5] and later improved by Assambo \& Burke [6]. The $46 \mathrm{~dB}$ of differential gain is split primarily between the first stage with $14.4 \mathrm{~dB}$ and the second stage with $25.6 \mathrm{~dB}$ while the third stage provides $6 \mathrm{~dB}$ of the gain with differential-tosingle-ended conversion. The input stage provides a high differential-mode and common-mode impedance of $100 \mathrm{M} \Omega$ in order to preserve adequate common-moderejection-ratio (CMRR) when interfacing with highimpedance dry electrodes. The amplifier operates from a single $3.3 \mathrm{~V}$ supply rail and the input stages are biased to a mid-rail voltage of $1.65 \mathrm{~V}$ using a separate dc-to-dc convertor chip not shown in the schematic. ESD protection and r.f. suppression elements are also omitted for clarity. The operational amplifier used in this design is the AD8648 (Analogue Devices Inc.) which is a quad amplifier package with each op-amp having a gainbandwidth product of $24 \mathrm{Mz}$ and a quiescent current of $2 \mathrm{~mA}$. It also offers low offsets and noise and a CMRR of $84 \mathrm{~dB}$. The input impedance of each amplifier is unspecified, however.

\subsection{Electrodes}

The electrodes used in the Moyo unit shown in Figure 1 are made of stainless steel with a matt-finish surface, and are easily disinfected before use with an alcohol wipe. Electrical contact is made with the electrodes by the user gripping the unit in both hands with their fingers placed on the electrode surfaces. Only the firmness of the user's grip determines the contact pressure. In order to obtain an indication of the contact impedance of these electrodes a method formerly reported by Baba \& Burke [7] was used to characterize the electrodes. An equivalent electrical model of the electrode is shown in Figure 3. This can be seen to contain two parallel C-R networks as well as two series resistors. The frequency response of the model is therefore characterized by two time constants. The values of these time constants are very large, which makes their evaluation in the frequency domain very difficult and consequently this was done in the time domain instead.

The current source shown in Figure 4 was used to inject a minute current of $2 \mu \mathrm{A}$ through the electrodes while being held by the user. The current was activated and then deactivated via the relay for a stable period in the region of $30 \mathrm{~s}$. A $10 \mathrm{kHz}$ sinewave signal was also used to allow the high frequency purely resistive elements to be evaluated. A program in MATLAB (MathWorks Inc.) was then used to fit a two time constant C-R model shown in Figure 3 to the recorded voltage waveforms and to determine the values of the individual components of the model.

This was done for measurements made on 20 subjects of varying age, sex, nationality and skin colour, in order to establish the extent of the variation of electrode impedance to be expected. Only the passive components of the model were of interest in this instance and the $\mathrm{dc}$ polarization potentials were not measured. The ranges of values measured for each component of the model are listed in Table. 1 below. Plots of the magnitude and phase as functions of frequency of a single stainless steel electrode are shown in Figure 5. Plots are shown for a light grip on the electrode and for a falling current. The impedance varies considerably within the frequency range of the ECG signal. The magnitude falls off abruptly 


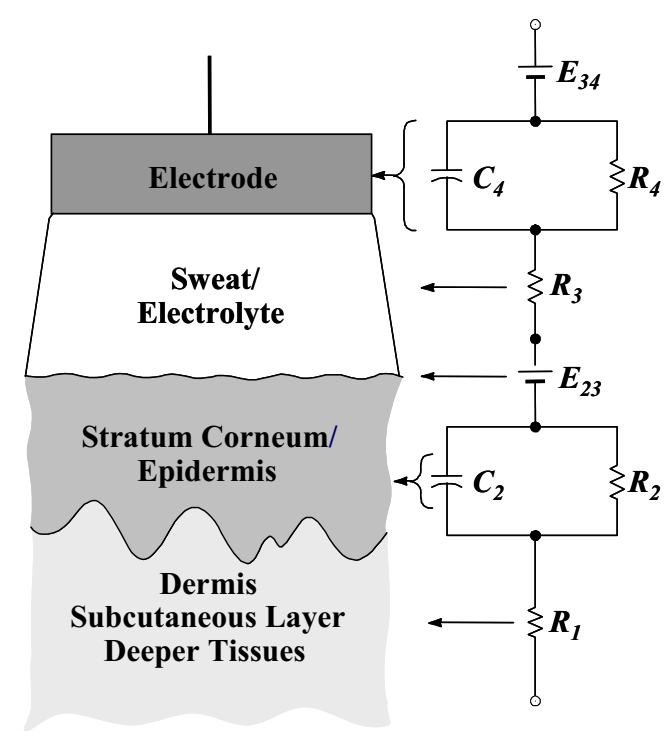

Figure 3. Equivalent Electrical Model of the Electrodes

above $1 \mathrm{~Hz}$ and the phase falls off above $10 \mathrm{~Hz}$. It can be seen that the magnitude of the impedance is less than $30 \mathrm{k} \Omega$ at frequencies above approximately $10 \mathrm{~Hz}$. This is considerably lower than the input common-mode resistance of the amplifier or of the protection resistor, $\mathrm{R}_{1}$, shown in the schematic diagram of the amplifier in Figure 2. This indicates that the signal levels involved in measuring the contact impedance of such electrodes are by nature quite low.

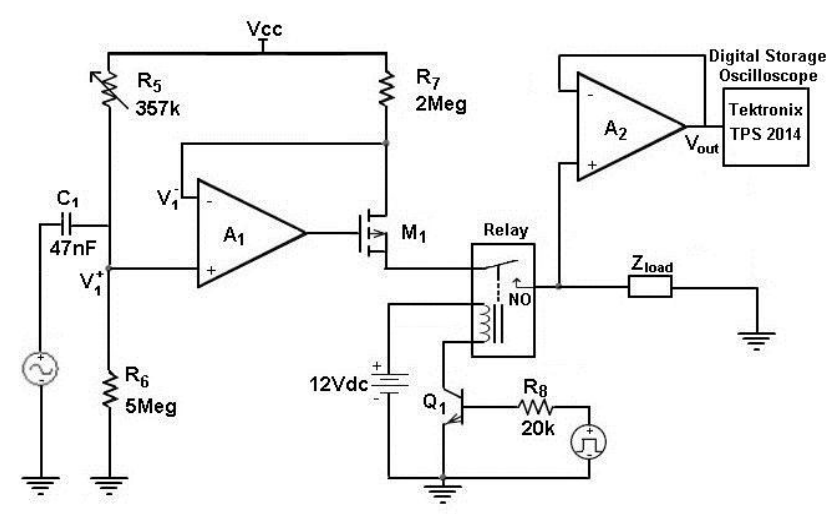

Figure 4. Electrode Impedance Measurement Circuit

Table 1. Range of Values for Electrode Model Elements

\begin{tabular}{|c|c|c|c|}
\hline Element & Minimum & Maximum & Unit \\
\hline $\mathrm{R}_{1}+\mathrm{R}_{3}$ & 20 & 45 & $\mathrm{k} \Omega$ \\
\hline $\mathrm{R}_{2}$ & 2.03 & 331 & $\mathrm{k} \Omega$ \\
\hline $\mathrm{C}_{2}$ & 0.000619 & 891 & $\mu \mathrm{F}$ \\
\hline $\mathrm{R}_{4}$ & 1.96 & 911 & $\mathrm{k} \Omega$ \\
\hline $\mathrm{C}_{4}$ & 0.00134 & 976 & $\mu \mathrm{F}$ \\
\hline$\tau_{2}$ & 0.0000227 & 25.4 & $\mathrm{~s}$ \\
\hline$\tau_{4}$ & 0.00039 & 889 & $\mathrm{~s}$ \\
\hline
\end{tabular}

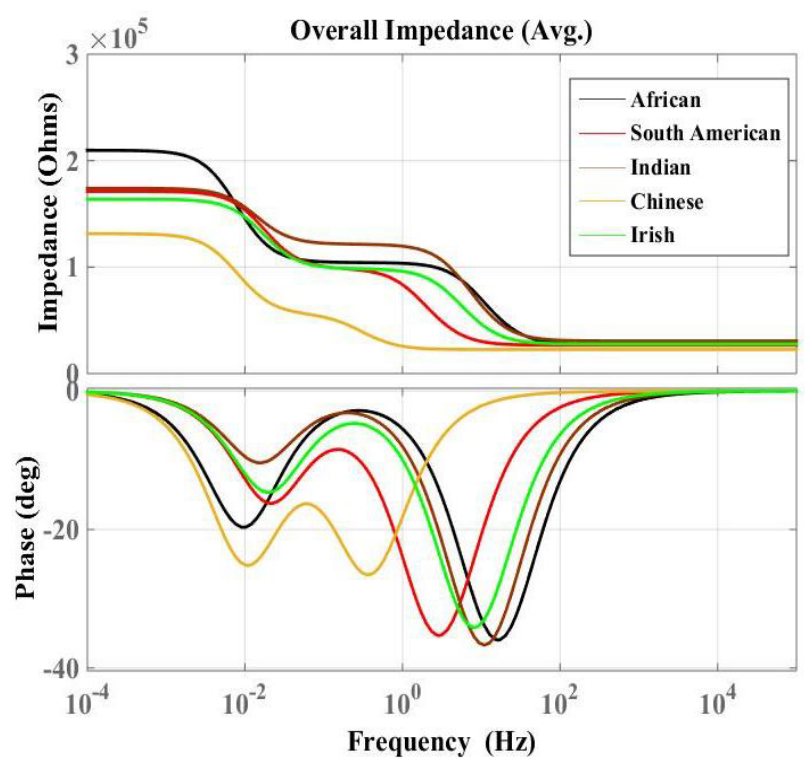

Figure 5. Impedance of Blasted Stainless Steel Electrodes

\section{Methodology}

A block diagram of the electrode contact monitoring system is shown in Figure 6. An oscillator and bandpass filter are used to provide a sinusoidal source signal for measurement purposes that is outside of the range of the ECG signal spectrum. This signal is then buffered in unity-gain inverting and non-inverting amplifiers to provide antiphase measurement sources. These antiphase signals are then fed through the common-mode input resistors of the ECG amplifier to the electrodes. The first stage of the ECG amplifier is also used to provide initial gain for the contact monitoring signal. Following this, bandpass filters are used to extract the sinusoidal signal from the ECG signal at the outputs of the first stage of the amplifier. The output signal of the upper and lower bandpass filters are then fed into separate half-wave rectifiers in order to provide a steady-state indication of the contact impedance at each electrode. The output voltages of the rectifiers are then compared with reference levels in separate threshold detectors in each channel. When the contact impedance of an electrode exceeds the reference value, the output of the corresponding threshold detector goes high. This output is then used to drive a LED via a suitable buffer circuit. LEDs were used to provide visual alarms in the prototype unit but this will implemented on the LCD display in the final unit to maintain very low power consumption.

\section{Measurement Circuit}

\subsection{ECG amplifier modification}

A full schematic diagram of the electrode contact monitoring system and its incorporation into the ECG amplifier is shown in Figure 7. ESD protection diodes, $\mathrm{D}_{3}$ and $\mathrm{D}_{4}$ and r.f. suppression capacitors, $\mathrm{C}_{15}$, are also 


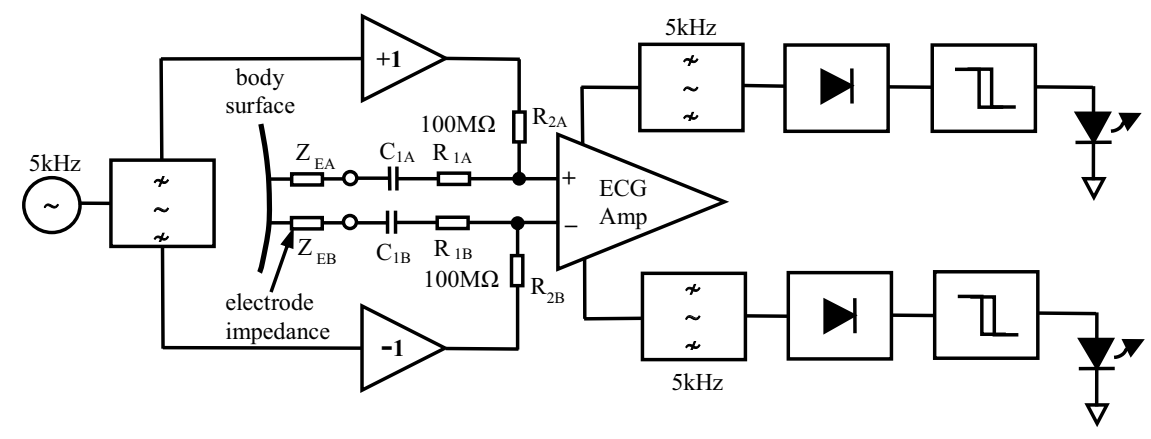

Figure 6. Block Diagram of the Electrode Impedance Measuremnt System

shown in each channel. It was decided to use the frontend stage of the ECG amplifier to provide the first phase of amplification for the electrode contact monitoring signal. The gains of the three stages of this amplifier were revised accordingly. The first stage gain was changed to $22 \mathrm{~dB}$, the second stage to $25 \mathrm{~dB}$ and the third stage was revised to give unity gain. The revised component values set the $3 \mathrm{~dB}$ lower cut-off frequency at $0.67 \mathrm{~Hz}$ as required by the international standards for clinical heart-rate monitoring [8]. It also increased the CMRR by $6 \mathrm{~dB}$ in the ECG signal bandwidth. The AD8619 (Analog Devices Inc.) op-amp was used in the first stage of the ECG amplifier as it has a gain-bandwidth product of $400 \mathrm{kHz}$ with a quiescent current of $38 \mu \mathrm{A}$ per channel. Its upper cut-off frequency was self-limited by the op-amp at greater than $10 \mathrm{kHz}$, thus allowing full amplification of the $5 \mathrm{kHz}$ contact monitoring signal. The OPA4379 (Texas Instruments Inc.) op-amp was used in the second and third amplifier stages as it provides a gain-bandwidth product of $90 \mathrm{kHz}$ with a quiescent current of only $3 \mu \mathrm{A}$ per channel. The upper cut-off frequencies of the second and third stages of the amplifier were set at $350 \mathrm{~Hz}$ and $480 \mathrm{~Hz}$ respectively. This reduced the power consumption of the redesigned ECG amplifier by a factor of 120 .

\subsection{Measurement signal generation}

An astable multivibrator built around the comparator $\mathrm{A}_{6}$ is used to generate a square-wave at a frequency of $5 \mathrm{kHz}$. The TLV3201 (Texas Instruments Inc.) is used as the comparator because of its high slew rate and rail-to-rail voltage swing with a low quiescent current of $40 \mu \mathrm{A}$. The bandpass filter centred around op-amps $A_{7}$ and $A_{8}$ is a multiple feedback configuration having a centre frequency of $5 \mathrm{kHz}$ and a Q-factor of 12 . This provides a sinusoidal source signal with an amplitude of $1.35 \mathrm{~V}$ peak. The choice of contact monitoring frequency was a design compromise. A high frequency well above the ECG bandwidth is desirable as it is easy to extract and separate from the wanted ECG signal. However, the parasitic input capacitance of operational amplifiers and r.f. suppression capacitors have a significant shunting effect on the electrode contact impedance at higher frequencies so that the signal developed across this impedance is very small. The design value of $5 \mathrm{kHz}$ was chosen to allow a reasonable signal level for measurement, while at the same time maintaining the measurement frequency at least a decade above the ECG band.

\subsection{Signal injection}

The sinewave is then passed through two buffer amplifiers, a non-inverting unity-gain stage built around op-amp $\mathrm{A}_{10}$ and a unity-gain inverting stage built around op-amp $\mathrm{A}_{11}$, which also have mid-rail bias voltages. The in-phase component is fed to the upper electrode point via the upper-channel common-mode input resistor $R_{2 A}$. The inverted component is fed to the lower electrode contact point via the lower-channel common-mode resistor $R_{2 B}$. Both resistors $R_{2 A}$ and $R_{2 B}$ of $100 M \Omega$ are bypassed by parallel capacitors of $1 \mathrm{pF}$ in order to lower the series impedance seen at the signal injection frequency of $5 \mathrm{kHz}$ to around $24 \mathrm{M} \Omega$. This method of feeding the contact monitoring signal to the electrodes preserves the high common-mode impedance at the ECG amplifier input and consequently the CMRR. The use of antiphase signals also allows the signal developed on the mother's body to be kept close to zero.

The levels of $5 \mathrm{kHz}$ signal present at the input terminals of the ECG amplifier are those developed across the contact impedances of each electrode namely, $Z_{\mathrm{EA}}$ and $Z_{\mathrm{EB}}$. With good contact and low skin-electrode impedance the signal levels are extremely small as the impedance of resistor $\mathrm{R}_{2}$ in parallel with capacitor $\mathrm{C}_{14}$ forms a potential divider with the electrode contact impedance, $Z_{\mathrm{E}}$, at each terminal of the amplifier. In fact, most of the $5 \mathrm{kHz}$ signal present is actually developed across the protection resistor $\mathrm{R}_{1}$ in each case. This has a value of $100 \mathrm{k} \Omega$, which is much greater than the typical contact impedance of the electrode itself.

\subsection{Signal extraction}

Under normal conditions with good electrode contact the signal at $5 \mathrm{kHz}$ present at the amplifier input terminals is of the order of $12 \mathrm{mV}$. On the other hand, when electrode contact is lost altogether, a signal level of just less than $125 \mathrm{mV}$ is obtained at the inputs terminals of the amplifier.

If a maximum electrode contact impedance is set at $100 \mathrm{k} \Omega$, which is the same value as the resistor, $R_{1}$, then the maximum expected signal with good electrode contact appearing at the input of the ECG amplifier is of the order of $22 \mathrm{mV}$. The gain of $22 \mathrm{~dB}$ in the first stage of the ECG amplifier provides the initial gain for the contact monitoring signal, raising the signal level at the output of this stage to $268 \mathrm{mV}$. 


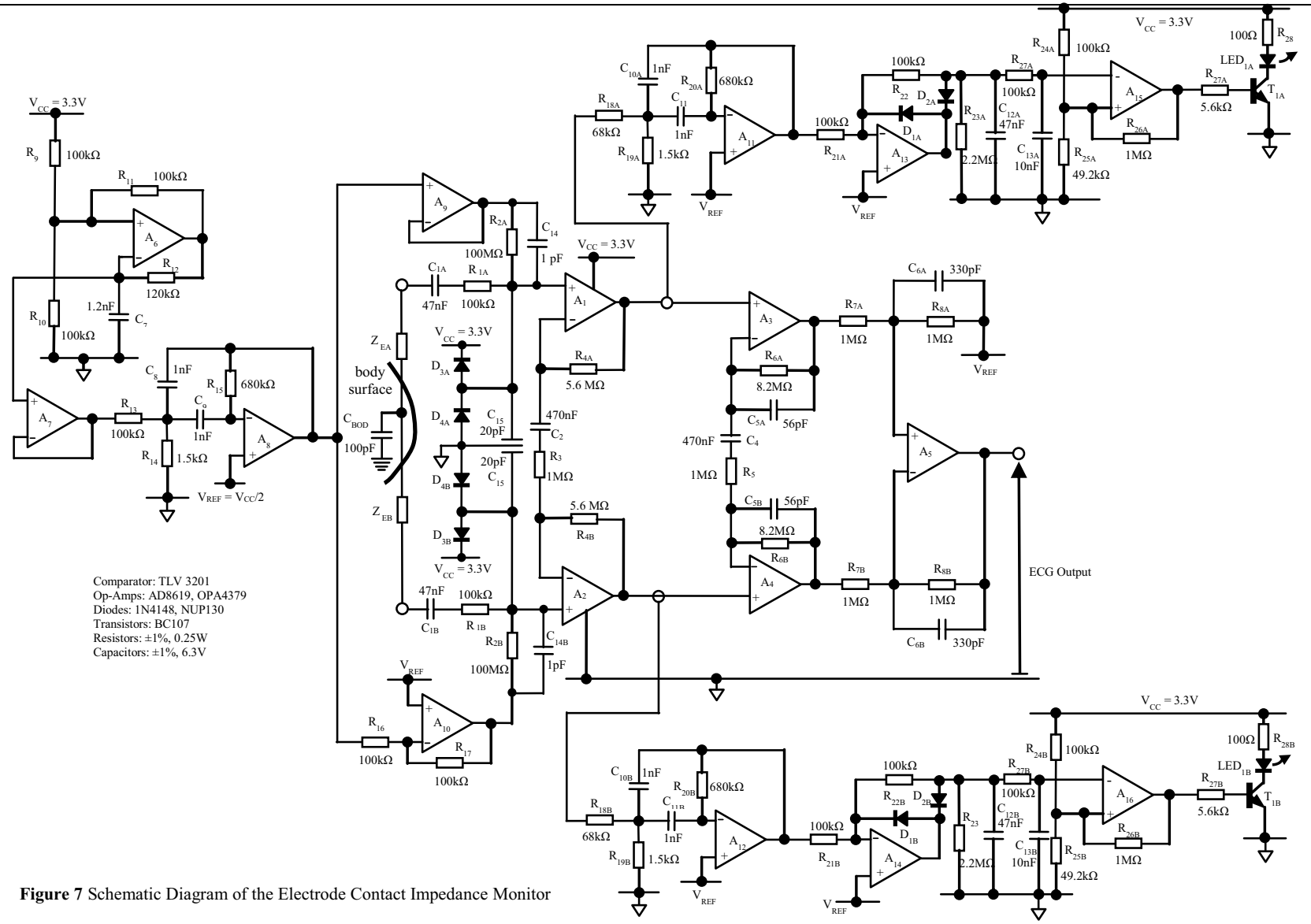

The electrode contact monitoring signals are extracted from the outputs of each side of the first stage of the ECG amplifier using band-pass filters built around op-amps $\mathrm{A}_{11}$ and $\mathrm{A}_{12}$. These filters are multiple feedback structures having a centre frequency of $5 \mathrm{kHz}$, a gain of $14 \mathrm{~dB}$ at this frequency and a Q-factor of 12 . All of the bandpass filters were implemented using the AD8619 op-amp. This provides an overall gain of $36 \mathrm{~dB}$ or a factor of 60 to the signal developed across the resistor $\mathrm{R}_{1}$ and the electrode impedance $Z_{E}$ in series. The output signal level from the filters with good electrode contact is then of the order of $350 \mathrm{mV}$. At the limit of poor electrode contact impedance of $100 \mathrm{k} \Omega$, this rises to approximately $660 \mathrm{mV}$ peak.

\subsection{Signal level detection}

The output sinewaves from the band-pass filters in each channel are then fed into precision half-wave rectifiers built around op-amps $\mathrm{A}_{13}$ and $\mathrm{A}_{14}$. The rectification process has unity gain but is inverting and includes envelope detection of the sinewave by means of a C-R smoothing circuit having a time-constant of $1 \mathrm{~s}$. This provides a steady-state signal level representing the amplitude of the recovered sinewave at $5 \mathrm{kHz}$. The rectified level is negative-going with respect to the reference voltage of half the supply, $\mathrm{V}_{\mathrm{REF}}=1.65 \mathrm{~V}$. Therefore, at the limit of poor electrode contact, the output voltage of op-amp $\mathrm{A}_{13}$ or $\mathrm{A}_{14}$ falls to $1 \mathrm{~V}$.

Finally, the rectified voltages in each channel are fed into threshold detectors built around op-amps $\mathrm{A}_{15}$ and
$\mathrm{A}_{16}$. The reference voltage of each threshold detector is set at $1.09 \mathrm{~V}$ relative to ground by resistors $R_{24}$ and $R_{25}$ with approximately $40 \mathrm{mV}$ of hysteresis added by the positive feedback provided by resistor $\mathrm{R}_{26}$. The action of the threshold detectors is also inverting. Consequently, when the rectified $5 \mathrm{kHz}$ signal level reaches a threshold of $700 \mathrm{mV}$ below $\mathrm{V}_{\mathrm{REF}}$ or $1.09 \mathrm{Vdc}$, the op-amp acting as a comparator changes state and its output goes from $0 \mathrm{~V}$ representing good electrode contact, to the supply voltage of $3.3 \mathrm{~V}$ representing poor electrode contact.

All of the op-amps used in the signal level assessment circuitry are AD8657s in order to minimise power consumption. This HI output voltage of the op-amp is then used to feed a bipolar transistor based light-emittingdiode (LED) driver that provides a current of $10 \mathrm{~mA}$ to the activated LED. The LED will be replaced in the final design by a segment on the LCD in the Moyo unit.

\section{Performance Verification}

For test purposes, the electrodes were implemented as matched resistors in each channel, in the first instance. The value of these resistors were varied between $10 \mathrm{k} \Omega$ and $10 \mathrm{M} \Omega$ and the signal level at the output of the filters, $\mathrm{V}_{\mathrm{A} 11}, \mathrm{~V}_{\mathrm{A} 12}$, the half-wave rectifiers, $\mathrm{V}_{\mathrm{R} 23 \mathrm{~A}}, \mathrm{~V}_{\mathrm{R} 23 \mathrm{~B}}$ and the threshold detectors, $\mathrm{V}_{\mathrm{A} 15}, \mathrm{~V}_{\mathrm{A} 16}$, recorded as shown in Table 2. The threshold detector logic outputs, change state at a value of electrode resistance between $100 \mathrm{k} \Omega$ and $200 \mathrm{k} \Omega$ as required. Imbalance in the electrode 
resistance in each channel was also investigated and the output logic states of the threshold detectors are shown in Table 3. An electrode impedance above the threshold level in either channel caused the threshold detectors in both channels to register this. This is due to crosscoupling in the differential input stage of the amplifier.

Table 3. Threshold Detector State vs. Electrode Resistance

\begin{tabular}{|c|c|c|c|c|}
\hline $\begin{array}{c}\text { Upper } \rightarrow \\
\text { Lower } \downarrow\end{array}$ & $10 \mathrm{k} \Omega$ & $100 \mathrm{k} \Omega$ & $200 \mathrm{k} \Omega$ & $10 \mathrm{M} \Omega$ \\
\hline $10 \mathrm{k} \Omega$ & $\mathrm{LO} / \mathrm{LO}$ & $\mathrm{LO} / \mathrm{LO}$ & $\mathrm{HI} / \mathrm{HI}$ & $\mathrm{HI} / \mathrm{HI}$ \\
\hline $100 \mathrm{k} \Omega$ & $\mathrm{LO} / \mathrm{LO}$ & $\mathrm{LO} / \mathrm{LO}$ & $\mathrm{HI} / \mathrm{HI}$ & $\mathrm{HI} / \mathrm{HI}$ \\
\hline $200 \mathrm{k} \Omega$ & $\mathrm{HI} / \mathrm{HI}$ & $\mathrm{HI} / \mathrm{HI}$ & $\mathrm{HI} / \mathrm{HI}$ & $\mathrm{HI} / \mathrm{HI}$ \\
\hline $10 \mathrm{M} \Omega$ & $\mathrm{HI} / \mathrm{HI}$ & $\mathrm{HI} / \mathrm{HI}$ & $\mathrm{HI} / \mathrm{HI}$ & $\mathrm{HI} / \mathrm{HI}$ \\
\hline
\end{tabular}

\section{References}

[1] M. J Burke, C. Molloy and H. Fossan "HighImpedance Electrode Contact Monitoring" Proc. $19^{\text {th }}$ WSEAS Int. Conf. Circuits, Sys., Comm. \& Comput., Zakynthos, Paper No. 581-6000, July 2015.

[2] http://www.saferbirths.com

[3] http://www.who.int/en
[4] M. M. Murray, "Maternal or Fetal Heart Rate? Avoiding Intrapartum Misidentification" J. Obstet. Gynecol. \& Neonatal Nurs., vol. 33, pp33-104, 2004.

[5] M. J. Burke and D. T. Gleeson "A Micropower Dryelectrode ECG Pre-amplifier" IEEE Trans. Biomed. Eng., vol. 47, pp155-162, 2000.

[6] C. Assambo and M. J. Burke, "An Improved VeryLow Power Preamplifier for Use with Ungelled Electrodes in ECG Recording” NAUN Int. J. Biol. \& Biomed. Eng., vol. 1, pp. 25-35, 2007.

[7] A. Baba and M. J. Burke, "Electrical Characterization of Dry Electrodes for ECG Recording" Proc. $12^{\text {th }}$ WSEAS Int. Conf. Circuits, Sys., Comms. \& Comput., Crete, Paper No. 591-226, July 2008.

[8] International Electrotechnical Commission, Medical electrical equipment Part 2-27: Particular requirements for the safety, including essential performance, of electrocardiographic monitoring equipment, IEC Std. IEC60 601-2-27: 3rd ed., 2011.

Acknowledgement: This work was supported by the Norwegian Research Council Grant No. ES506110 under the Safer Births Program.

Table 2. Voltage at Key Circuit Nodes vs. Electrode Resistance

\begin{tabular}{|c|c|c|c|c|c|c|c|c|c|c|}
\hline $\begin{array}{c}\mathrm{R}_{\mathrm{E}} \\
(\Omega)\end{array}$ & $\begin{array}{c}\mathrm{V}_{\mathrm{iA}} \\
(\mathrm{mV})\end{array}$ & $\begin{array}{c}\mathrm{V}_{\mathrm{iB}} \\
(\mathrm{mV})\end{array}$ & $\begin{array}{c}\mathrm{V}_{\mathrm{A} 1} \\
(\mathrm{mV})\end{array}$ & $\begin{array}{c}\mathrm{V}_{\mathrm{A} 2} \\
(\mathrm{mV})\end{array}$ & $\begin{array}{c}\mathrm{V}_{\mathrm{A} 11} \\
(\mathrm{~V})\end{array}$ & $\begin{array}{c}\mathrm{V}_{\mathrm{A} 12} \\
(\mathrm{~V})\end{array}$ & $\begin{array}{c}\mathrm{V}_{\mathrm{R} 27 \mathrm{~A}} \\
(\mathrm{~V})\end{array}$ & $\begin{array}{c}\mathrm{V}_{\mathrm{R} 27 \mathrm{~B}} \\
(\mathrm{~V})\end{array}$ & $\begin{array}{c}\mathrm{V}_{\mathrm{A} 15} \\
(\operatorname{logic})\end{array}$ & $\begin{array}{c}\mathrm{V}_{\mathrm{A} 16} \\
(\operatorname{logic})\end{array}$ \\
\hline $10 \mathrm{k}$ & 26 & 22 & 166 & 112 & 0.63 & 0.58 & 1.40 & 1.38 & $\mathrm{LO}$ & $\mathrm{LO}$ \\
\hline $100 \mathrm{k}$ & 40 & 25 & 285 & 226 & 0.88 & 0.81 & 1.24 & 1.27 & $\mathrm{LO}$ & $\mathrm{LO}$ \\
\hline $200 \mathrm{k}$ & 50 & 38 & 380 & 290 & 1.21 & 1.04 & 1.07 & 1.16 & $\mathrm{HI}$ & $\mathrm{HI}$ \\
\hline $10 \mathrm{M}$ & 64 & 54 & 608 & 588 & 2.68 & 2.12 & 0.51 & 0.73 & $\mathrm{HI}$ & $\mathrm{HI}$ \\
\hline
\end{tabular}

